\title{
Model Checking. Part II
}

\author{
Kazuhisa Ishida \\ Shinshu University \\ Nagano, Japan
}

\begin{abstract}
Summary. This article provides the definition of linear temporal logic (LTL) and its properties relevant to model checking based on [9]. Mizar formalization of LTL language and satisfiability is based on $[2,3]$.
\end{abstract}

MML identifier: MODELC_2, version: $\underline{7.9 .01 \quad 4.101 .1015}$

The articles [8], [11], [6], [5], [7], [1], [4], [12], and [10] provide the notation and terminology for this paper.

Let $x$ be a set. The functor CastNat $x$ yielding a natural number is defined by:

(Def. 1) CastNat $x=\left\{\begin{array}{l}x, \text { if } x \text { is a natural number, } \\ 0, \text { otherwise. }\end{array}\right.$

Let $W_{1}$ be a set. A sequence of $W_{1}$ is a function from $\mathbb{N}$ into $W_{1}$.

For simplicity, we adopt the following rules: $k, n$ denote natural numbers, $a$ denotes a set, $D, S$ denote non empty sets, and $p, q$ denote finite sequences of elements of $\mathbb{N}$.

Let us consider $n$. The functor atom. $n$ yielding a finite sequence of elements of $\mathbb{N}$ is defined as follows:

(Def. 2) atom. $n=\langle 6+n\rangle$.

Let us consider $p$. The functor $\neg p$ yielding a finite sequence of elements of $\mathbb{N}$ is defined by:

(Def. 3) $\neg p=\langle 0\rangle^{\wedge} p$.

Let us consider $q$. The functor $p \wedge q$ yields a finite sequence of elements of $\mathbb{N}$ and is defined by:

(Def. 4) $p \wedge q=\langle 1\rangle^{\frown} p^{\frown} q$.

The functor $p \vee q$ yielding a finite sequence of elements of $\mathbb{N}$ is defined by: 
(Def. 5) $\quad p \vee q=\langle 2\rangle\rangle^{\frown} p^{\frown} q$.

Let us consider $p$. The functor $\mathcal{X} p$ yielding a finite sequence of elements of $\mathbb{N}$ is defined as follows:

(Def. 6) $\mathcal{X} p=\langle 3\rangle^{\frown} p$.

Let us consider $q$. The functor $p \mathcal{U} q$ yielding a finite sequence of elements of $\mathbb{N}$ is defined by:

(Def. 7) $p \mathcal{U} q=\langle 4\rangle{ }^{\wedge} p^{\frown} q$.

The functor $p \mathcal{R} q$ yields a finite sequence of elements of $\mathbb{N}$ and is defined as follows:

(Def. 8) $p \mathcal{R} q=\langle 5\urcorner^{\wedge} p^{\frown} q$.

The non empty set $\mathrm{WFF}_{\text {LTL }}$ is defined by the conditions (Def. 9).

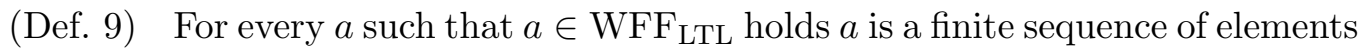
of $\mathbb{N}$ and for every $n$ holds atom. $n \in \mathrm{WFF}_{\text {LTL }}$ and for every $p$ such that $p \in \mathrm{WFF}_{\mathrm{LTL}}$ holds $\neg p \in \mathrm{WFF}_{\mathrm{LTL}}$ and for all $p, q$ such that $p, q \in \mathrm{WFF}_{\mathrm{LTL}}$ holds $p \wedge q \in \mathrm{WFF}_{\mathrm{LTL}}$ and for all $p, q$ such that $p, q \in \mathrm{WFF}_{\mathrm{LTL}}$ holds $p \vee q \in \mathrm{WFF}_{\text {LTL }}$ and for every $p$ such that $p \in \mathrm{WFF}_{\text {LTL }}$ holds $\mathcal{X} p \in$ $\mathrm{WFF}_{\mathrm{LTL}}$ and for all $p, q$ such that $p, q \in \mathrm{WFF}_{\mathrm{LTL}}$ holds $p \mathcal{U} q \in \mathrm{WFF}_{\mathrm{LTL}}$ and for all $p, q$ such that $p, q \in \mathrm{WFF}_{\mathrm{LTL}}$ holds $p \mathcal{R} q \in \mathrm{WFF}_{\mathrm{LTL}}$ and for every $D$ such that for every $a$ such that $a \in D$ holds $a$ is a finite sequence of elements of $\mathbb{N}$ and for every $n$ holds atom. $n \in D$ and for every $p$ such that $p \in D$ holds $\neg p \in D$ and for all $p, q$ such that $p, q \in D$ holds $p \wedge q \in D$ and for all $p, q$ such that $p, q \in D$ holds $p \vee q \in D$ and for every $p$ such that $p \in D$ holds $\mathcal{X} p \in D$ and for all $p, q$ such that $p, q \in D$ holds $p \mathcal{U} q \in D$ and for all $p, q$ such that $p, q \in D$ holds $p \mathcal{R} q \in D$ holds $\mathrm{WFF}_{\text {LTL }} \subseteq D$.

Let $I_{1}$ be a finite sequence of elements of $\mathbb{N}$. We say that $I_{1}$ is LTL-formulalike if and only if:

(Def. 10) $I_{1}$ is an element of $\mathrm{WFF}_{\mathrm{LTL}}$.

Let us observe that there exists a finite sequence of elements of $\mathbb{N}$ which is LTL-formula-like.

An LTL-formula is a LTL-formula-like finite sequence of elements of $\mathbb{N}$.

Next we state the proposition

(1) $a$ is an LTL-formula iff $a \in \mathrm{WFF}_{\mathrm{LTL}}$.

In the sequel $F, F_{1}, G, H, H_{1}, H_{2}$ denote LTL-formulae.

Let us consider $n$. Observe that atom. $n$ is LTL-formula-like.

Let us consider $H$. Note that $\neg H$ is LTL-formula-like and $\mathcal{X} H$ is LTLformula-like. Let us consider $G$. One can check the following observations:

* $H \wedge G$ is LTL-formula-like,

* $H \vee G$ is LTL-formula-like,

* $H \mathcal{U} G$ is LTL-formula-like, and 
* $\quad H \mathcal{R} G$ is LTL-formula-like.

Let us consider $H$. We say that $H$ is atomic if and only if:

(Def. 11) There exists $n$ such that $H=$ atom. $n$.

We say that $H$ is negative if and only if:

(Def. 12) There exists $H_{1}$ such that $H=\neg H_{1}$.

We say that $H$ is conjunctive if and only if:

(Def. 13) There exist $F, G$ such that $H=F \wedge G$.

We say that $H$ is disjunctive if and only if:

(Def. 14) There exist $F, G$ such that $H=F \vee G$.

We say that $H$ has next operator if and only if:

(Def. 15) There exists $H_{1}$ such that $H=\mathcal{X} H_{1}$.

We say that $H$ has until operator if and only if:

(Def. 16) There exist $F, G$ such that $H=F \mathcal{U} G$.

We say that $H$ has release operator if and only if:

(Def. 17) There exist $F, G$ such that $H=F \mathcal{R} G$.

Next we state two propositions:

(2) $H$ is either atomic, or negative, or conjunctive, or disjunctive, or has next operator, or until operator, or release operator.

(3) $1 \leq$ len $H$.

Let us consider $H$. Let us assume that $H$ is either negative or has next operator. The functor $\operatorname{Arg}(H)$ yields an LTL-formula and is defined by:

(Def. 18)(i) $\neg \operatorname{Arg}(H)=H$ if $H$ is negative,

(ii) $\mathcal{X} \operatorname{Arg}(H)=H$, otherwise.

Let us consider $H$. Let us assume that $H$ is either conjunctive or disjunctive or has until operator or release operator. The functor $\operatorname{Left} \operatorname{Arg}(H)$ yielding an LTL-formula is defined as follows:

(Def. 19)(i) There exists $H_{1}$ such that $\operatorname{Left} \operatorname{Arg}(H) \wedge H_{1}=H$ if $H$ is conjunctive,

(ii) there exists $H_{1}$ such that $\operatorname{Left} \operatorname{Arg}(H) \vee H_{1}=H$ if $H$ is disjunctive,

(iii) there exists $H_{1}$ such that $\operatorname{Left} \operatorname{Arg}(H) \mathcal{U} H_{1}=H$ if $H$ has until operator,

(iv) there exists $H_{1}$ such that $\operatorname{Left} \operatorname{Arg}(H) \mathcal{R} H_{1}=H$, otherwise.

The functor $\operatorname{Right} \operatorname{Arg}(H)$ yields an LTL-formula and is defined by:

(Def. 20)(i) There exists $H_{1}$ such that $H_{1} \wedge \operatorname{Right} \operatorname{Arg}(H)=H$ if $H$ is conjunctive,

(ii) there exists $H_{1}$ such that $H_{1} \vee \operatorname{Right} \operatorname{Arg}(H)=H$ if $H$ is disjunctive,

(iii) there exists $H_{1}$ such that $H_{1} \mathcal{U} \operatorname{Right} \operatorname{Arg}(H)=H$ if $H$ has until operator,

(iv) there exists $H_{1}$ such that $H_{1} \mathcal{R} \operatorname{Right} \operatorname{Arg}(H)=H$, otherwise.

The following propositions are true:

(4) If $H$ is negative, then $H=\neg \operatorname{Arg}(H)$. 
(5) If $H$ has next operator, then $H=\mathcal{X} \operatorname{Arg}(H)$.

(6) If $H$ is conjunctive, then $H=\operatorname{Left} \operatorname{Arg}(H) \wedge \operatorname{Right} \operatorname{Arg}(H)$.

(7) If $H$ is disjunctive, then $H=\operatorname{Left} \operatorname{Arg}(H) \vee \operatorname{Right} \operatorname{Arg}(H)$.

(8) If $H$ has until operator, then $H=\operatorname{Left} \operatorname{Arg}(H) \mathcal{U} \operatorname{Right} \operatorname{Arg}(H)$.

(9) If $H$ has release operator, then $H=\operatorname{Left} \operatorname{Arg}(H) \mathcal{R} \operatorname{Right} \operatorname{Arg}(H)$.

(10) If $H$ is either negative or has next operator, then len $H=1+\operatorname{len} \operatorname{Arg}(H)$ and len $\operatorname{Arg}(H)<$ len $H$.

(11) Suppose $H$ is either conjunctive or disjunctive or has until operator or release operator. Then len $H=1+$ len $\operatorname{Left} \operatorname{Arg}(H)+\operatorname{len} \operatorname{Right} \operatorname{Arg}(H)$ and len $\operatorname{Left} \operatorname{Arg}(H)<$ len $H$ and len $\operatorname{Right} \operatorname{Arg}(H)<$ len $H$.

Let us consider $H, F$. We say that $H$ is an immediate constituent of $F$ if and only if:

(Def. 21) $\quad F=\neg H$ or $F=\mathcal{X} H$ or there exists $H_{1}$ such that $F=H \wedge H_{1}$ or $F=H_{1} \wedge H$ or $F=H \vee H_{1}$ or $F=H_{1} \vee H$ or $F=H \mathcal{U} H_{1}$ or $F=H_{1} \mathcal{U} H$ or $F=H \mathcal{R} H_{1}$ or $F=H_{1} \mathcal{R} H$.

We now state a number of propositions:

(12) For all $F, G$ holds $(\neg F)(1)=0$ and $(F \wedge G)(1)=1$ and $(F \vee G)(1)=2$ and $(\mathcal{X} F)(1)=3$ and $(F \mathcal{U} G)(1)=4$ and $(F \mathcal{R} G)(1)=5$.

(13) $H$ is an immediate constituent of $\neg F$ iff $H=F$.

(14) $\quad H$ is an immediate constituent of $\mathcal{X} F$ iff $H=F$.

(15) $H$ is an immediate constituent of $F \wedge G$ iff $H=F$ or $H=G$.

(16) $H$ is an immediate constituent of $F \vee G$ iff $H=F$ or $H=G$.

(17) $H$ is an immediate constituent of $F \mathcal{U} G$ iff $H=F$ or $H=G$.

(18) $H$ is an immediate constituent of $F \mathcal{R} G$ iff $H=F$ or $H=G$.

(19) If $F$ is atomic, then $H$ is not an immediate constituent of $F$.

(20) If $F$ is negative, then $H$ is an immediate constituent of $F$ iff $H=\operatorname{Arg}(F)$.

(21) If $F$ has next operator, then $H$ is an immediate constituent of $F$ iff $H=\operatorname{Arg}(F)$.

(22) If $F$ is conjunctive, then $H$ is an immediate constituent of $F$ iff $H=$ $\operatorname{Left} \operatorname{Arg}(F)$ or $H=\operatorname{Right} \operatorname{Arg}(F)$.

(23) If $F$ is disjunctive, then $H$ is an immediate constituent of $F$ iff $H=$ $\operatorname{Left} \operatorname{Arg}(F)$ or $H=\operatorname{Right} \operatorname{Arg}(F)$.

(24) If $F$ has until operator, then $H$ is an immediate constituent of $F$ iff $H=\operatorname{LeftArg}(F)$ or $H=\operatorname{Right} \operatorname{Arg}(F)$.

(25) If $F$ has release operator, then $H$ is an immediate constituent of $F$ iff $H=\operatorname{Left} \operatorname{Arg}(F)$ or $H=\operatorname{Right} \operatorname{Arg}(F)$.

(26) Suppose $H$ is an immediate constituent of $F$. Then $F$ is either negative, or conjunctive, or disjunctive, or has next operator, or until operator, or 
release operator.

In the sequel $L$ denotes a finite sequence.

Let us consider $H, F$. We say that $H$ is a subformula of $F$ if and only if the condition (Def. 22) is satisfied.

(Def. 22) There exist $n, L$ such that

(i) $1 \leq n$,

(ii) $\operatorname{len} L=n$,

(iii) $L(1)=H$,

(iv) $L(n)=F$, and

(v) for every $k$ such that $1 \leq k<n$ there exist $H_{1}, F_{1}$ such that $L(k)=H_{1}$ and $L(k+1)=F_{1}$ and $H_{1}$ is an immediate constituent of $F_{1}$.

We now state the proposition

(27) $H$ is a subformula of $H$.

Let us consider $H, F$. We say that $H$ is a proper subformula of $F$ if and only if:

(Def. 23) $H$ is a subformula of $F$ and $H \neq F$.

One can prove the following propositions:

(28) If $H$ is an immediate constituent of $F$, then len $H<\operatorname{len} F$.

(29) If $H$ is an immediate constituent of $F$, then $H$ is a proper subformula of $F$.

(30) If $G$ is either negative or has next operator, then $\operatorname{Arg}(G)$ is a subformula of $G$.

(31) Suppose $G$ is either conjunctive or disjunctive or has until operator or release operator. Then $\operatorname{Left} \operatorname{Arg}(G)$ is a subformula of $G$ and $\operatorname{Right} \operatorname{Arg}(G)$ is a subformula of $G$.

(32) If $H$ is a proper subformula of $F$, then len $H<\operatorname{len} F$.

(33) If $H$ is a proper subformula of $F$, then there exists $G$ which is an immediate constituent of $F$.

(34) If $F$ is a proper subformula of $G$ and $G$ is a proper subformula of $H$, then $F$ is a proper subformula of $H$.

(35) If $F$ is a subformula of $G$ and $G$ is a subformula of $H$, then $F$ is a subformula of $H$.

(36) If $G$ is a subformula of $H$ and $H$ is a subformula of $G$, then $G=H$.

(37) If $G$ is either negative or has next operator and $F$ is a proper subformula of $G$, then $F$ is a subformula of $\operatorname{Arg}(G)$.

(38) Suppose that

(i) $G$ is either conjunctive or disjunctive or has until operator or release operator, and

(ii) $F$ is a proper subformula of $G$. 
Then $F$ is a subformula of $\operatorname{Left} \operatorname{Arg}(G)$ or a subformula of $\operatorname{Right} \operatorname{Arg}(G)$.

(39) If $F$ is a proper subformula of $\neg H$, then $F$ is a subformula of $H$.

(40) If $F$ is a proper subformula of $\mathcal{X} H$, then $F$ is a subformula of $H$.

(41) If $F$ is a proper subformula of $G \wedge H$, then $F$ is a subformula of $G$ or a subformula of $H$.

(42) If $F$ is a proper subformula of $G \vee H$, then $F$ is a subformula of $G$ or a subformula of $H$.

(43) If $F$ is a proper subformula of $G \mathcal{U} H$, then $F$ is a subformula of $G$ or a subformula of $H$.

(44) If $F$ is a proper subformula of $G \mathcal{R} H$, then $F$ is a subformula of $G$ or a subformula of $H$.

Let us consider $H$. The functor Subformulae $H$ yields a set and is defined by:

(Def. 24) $\quad a \in$ Subformulae $H$ iff there exists $F$ such that $F=a$ and $F$ is a subformula of $H$.

One can prove the following proposition

(45) $G \in$ Subformulae $H$ iff $G$ is a subformula of $H$.

Let us consider $H$. Observe that Subformulae $H$ is non empty.

Next we state two propositions:

(46) If $F$ is a subformula of $H$, then Subformulae $F \subseteq$ Subformulae $H$.

(47) If $a$ is a subset of Subformulae $H$, then $a$ is a subset of $\mathrm{WFF}_{\mathrm{LTL}}$.

In this article we present several logical schemes. The scheme LTLInd concerns a unary predicate $\mathcal{P}$, and states that:

For every $H$ holds $\mathcal{P}[H]$

provided the following conditions are satisfied:

- For every $H$ such that $H$ is atomic holds $\mathcal{P}[H]$,

- For every $H$ such that $H$ is either negative or has next operator and $\mathcal{P}[\operatorname{Arg}(H)]$ holds $\mathcal{P}[H]$, and

- Let given $H$. Suppose $H$ is either conjunctive or disjunctive or has until operator or release operator and $\mathcal{P}[\operatorname{Left} \operatorname{Arg}(H)]$ and $\mathcal{P}[\operatorname{RightArg}(H)]$. Then $\mathcal{P}[H]$.

The scheme LTLCompInd concerns a unary predicate $\mathcal{P}$, and states that:

For every $H$ holds $\mathcal{P}[H]$

provided the following condition is met:

- For every $H$ such that for every $F$ such that $F$ is a proper subformula of $H$ holds $\mathcal{P}[F]$ holds $\mathcal{P}[H]$.

Let $x$ be a set. The functor Cast LTL $x$ yielding an LTL-formula is defined by:

(Def. 25) Cast $_{\mathrm{LTL}} x=\left\{\begin{array}{l}x, \text { if } x \in \mathrm{WFF}_{\mathrm{LTL}}, \\ \text { atom. } 0, \text { otherwise. }\end{array}\right.$ 
We introduce LTL-model structures which are systems

〈 assignations, basic assignations, a conjunction, a disjunction, a negation, a next-operation, an until-operation, a release-operation $\rangle$, where the assignations constitute a non empty set, the basic assignations constitute a non empty subset of the assignations, the conjunction is a binary operation on the assignations, the disjunction is a binary operation on the assignations, the negation is a unary operation on the assignations, the next-operation is a unary operation on the assignations, the until-operation is a binary operation on the assignations, and the release-operation is a binary operation on the assignations.

Let $V$ be an LTL-model structure. An assignation of $V$ is an element of the assignations of $V$.

The subset atomic LTL $_{\text {of }} \mathrm{WFF}_{\mathrm{LTL}}$ is defined by:

(Def. 26) atomic $_{\mathrm{LTL}}=\{x ; x$ ranges over LTL-formulae: $x$ is atomic $\}$.

Let $V$ be an LTL-model structure, let $K_{1}$ be a function from atomic LTL $_{\text {L }}$ into the basic assignations of $V$, and let $f$ be a function from $\mathrm{WFF}_{\mathrm{LTL}}$ into the assignations of $V$. We say that $f$ is an evaluation for $K_{1}$ if and only if the condition (Def. 27) is satisfied.

(Def. 27) Let $H$ be an LTL-formula. Then

(i) if $H$ is atomic, then $f(H)=K_{1}(H)$,

(ii) if $H$ is negative, then $f(H)=($ the negation of $V)(f(\operatorname{Arg}(H)))$,

(iii) if $H$ is conjunctive, then $f(H)=($ the conjunction of $V)(f(\operatorname{Left} \operatorname{Arg}(H))$, $f(\operatorname{Right} \operatorname{Arg}(H)))$,

(iv) if $H$ is disjunctive, then $f(H)=($ the disjunction of $V)(f(\operatorname{Left} \operatorname{Arg}(H))$, $f(\operatorname{Right} \operatorname{Arg}(H)))$,

(v) if $H$ has next operator, then $f(H)=$ (the next-operation of $V)(f(\operatorname{Arg}(H)))$,

(vi) if $H$ has until operator, then $f(H)=$ (the until-operation of $V)(f(\operatorname{Left} \operatorname{Arg}(H)), f(\operatorname{Right} \operatorname{Arg}(H)))$, and

(vii) if $H$ has release operator, then $f(H)=$ (the release-operation of $V)(f(\operatorname{Left} \operatorname{Arg}(H)), f(\operatorname{Right} \operatorname{Arg}(H)))$.

Let $V$ be an LTL-model structure, let $K_{1}$ be a function from atomic LTL $_{\text {LT }}$ into the basic assignations of $V$, let $f$ be a function from $\mathrm{WFF}_{\mathrm{LTL}}$ into the assignations of $V$, and let $n$ be a natural number. We say that $f$ is a $n$-preevaluation for $K_{1}$ if and only if the condition (Def. 28) is satisfied.

(Def. 28) Let $H$ be an LTL-formula such that len $H \leq n$. Then

(i) if $H$ is atomic, then $f(H)=K_{1}(H)$,

(ii) if $H$ is negative, then $f(H)=($ the negation of $V)(f(\operatorname{Arg}(H)))$,

(iii) if $H$ is conjunctive, then $f(H)=($ the conjunction of $V)(f(\operatorname{Left} \operatorname{Arg}(H))$, $f(\operatorname{Right} \operatorname{Arg}(H)))$, 
(iv) if $H$ is disjunctive, then $f(H)=($ the disjunction of $V)(f(\operatorname{Left} \operatorname{Arg}(H))$, $f(\operatorname{Right} \operatorname{Arg}(H)))$,

(v) if $H$ has next operator, then $f(H)=$ (the next-operation of $V)(f(\operatorname{Arg}(H)))$,

(vi) if $H$ has until operator, then $f(H)=$ (the until-operation of $V)(f(\operatorname{Left} \operatorname{Arg}(H)), f(\operatorname{Right} \operatorname{Arg}(H)))$, and

(vii) if $H$ has release operator, then $f(H)=$ (the release-operation of $V)(f(\operatorname{Left} \operatorname{Arg}(H)), f(\operatorname{Right} \operatorname{Arg}(H)))$.

Let $V$ be an LTL-model structure, let $K_{1}$ be a function from atomic into the basic assignations of $V$, let $f, h$ be functions from $\mathrm{WFF}_{\mathrm{LTL}}$ into the assignations of $V$, let $n$ be a natural number, and let $H$ be an LTL-formula. The functor GraftEval $\left(V, K_{1}, f, h, n, H\right)$ yields a set and is defined by:

(Def. 29) GraftEval $\left(V, K_{1}, f, h, n, H\right)$

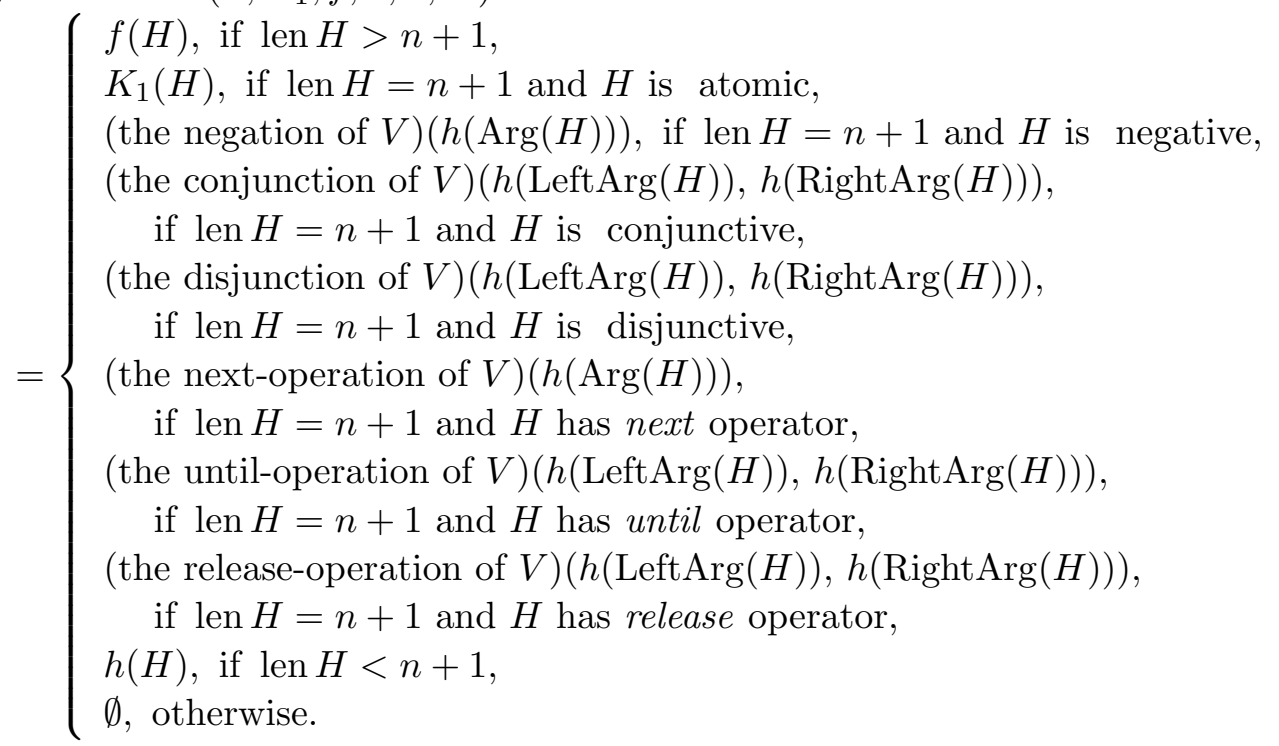

We adopt the following convention: $V$ denotes an LTL-model structure, $K_{1}$

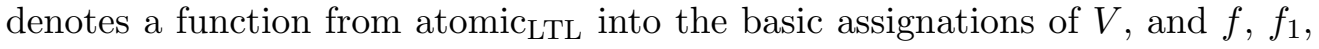
$f_{2}$ denote functions from $\mathrm{WFF}_{\mathrm{LTL}}$ into the assignations of $V$.

Let $V$ be an LTL-model structure, let $K_{1}$ be a function from atomic LTL $_{\text {L }}$ into the basic assignations of $V$, and let $n$ be a natural number. The functor EvalSet $\left(V, K_{1}, n\right)$ yields a non empty set and is defined by:

(Def. 30) $\operatorname{EvalSet}\left(V, K_{1}, n\right)=\left\{h ; h\right.$ ranges over functions from $\mathrm{WFF}_{\mathrm{LTL}}$ into the assignations of $V: h$ is a $n$-pre-evaluation for $\left.K_{1}\right\}$.

Let $V$ be an LTL-model structure, let $v_{0}$ be an element of the assignations of $V$, and let $x$ be a set. The functor $\operatorname{CastEval}\left(V, x, v_{0}\right)$ yielding a function from $\mathrm{WFF}_{\mathrm{LTL}}$ into the assignations of $V$ is defined by:

(Def. 31) $\operatorname{CastEval}\left(V, x, v_{0}\right)=\left\{\begin{array}{l}x, \text { if } x \in(\text { the assignations of } V)^{\mathrm{WFF}_{\mathrm{LTL}}}, \\ \mathrm{WFF}_{\mathrm{LTL}} \longmapsto v_{0}, \text { otherwise. }\end{array}\right.$ 
Let $V$ be an LTL-model structure and let $K_{1}$ be a function from atomic LTL $_{\text {L }}$ into the basic assignations of $V$. The functor EvalFamily $\left(V, K_{1}\right)$ yielding a non empty set is defined by the condition (Def. 32).

(Def. 32) Let $p$ be a set. Then $p \in \operatorname{EvalFamily}\left(V, K_{1}\right)$ if and only if the following conditions are satisfied:

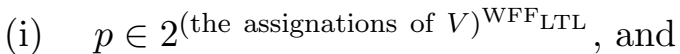

(ii) there exists a natural number $n$ such that $p=\operatorname{EvalSet}\left(V, K_{1}, n\right)$.

We now state two propositions:

(48) There exists $f$ which is an evaluation for $K_{1}$.

(49) If $f_{1}$ is an evaluation for $K_{1}$ and $f_{2}$ is an evaluation for $K_{1}$, then $f_{1}=f_{2}$.

Let $V$ be an LTL-model structure, let $K_{1}$ be a function from atomic LTL $_{2}$ into the basic assignations of $V$, and let $H$ be an LTL-formula. The functor Evaluate $\left(H, K_{1}\right)$ yields an assignation of $V$ and is defined by:

(Def. 33) There exists a function $f$ from $\mathrm{WFF}_{\mathrm{LTL}}$ into the assignations of $V$ such that $f$ is an evaluation for $K_{1}$ and Evaluate $\left(H, K_{1}\right)=f(H)$.

Let $V$ be an LTL-model structure and let $f$ be an assignation of $V$. The functor $\neg f$ yielding an assignation of $V$ is defined by:

(Def. 34) $\neg f=$ (the negation of $V)(f)$.

Let $V$ be an LTL-model structure and let $f, g$ be assignations of $V$. The functor $f \wedge g$ yields an assignation of $V$ and is defined by:

(Def. 35) $f \wedge g=($ the conjunction of $V)(f, g)$.

The functor $f \vee g$ yields an assignation of $V$ and is defined as follows:

(Def. 36) $\quad f \vee g=($ the disjunction of $V)(f, g)$.

Let $V$ be an LTL-model structure and let $f$ be an assignation of $V$. The functor $\mathcal{X} f$ yielding an assignation of $V$ is defined by:

(Def. 37) $\mathcal{X} f=($ the next-operation of $V)(f)$.

Let $V$ be an LTL-model structure and let $f, g$ be assignations of $V$. The functor $f \mathcal{U} g$ yielding an assignation of $V$ is defined by:

(Def. 38) $\quad f \mathcal{U} g=($ the until-operation of $V)(f, g)$.

The functor $f \mathcal{R} g$ yields an assignation of $V$ and is defined as follows:

(Def. 39) $\quad f \mathcal{R} g=$ (the release-operation of $V)(f, g)$.

One can prove the following propositions:

(50) $\operatorname{Evaluate}\left(\neg H, K_{1}\right)=\neg \operatorname{Evaluate}\left(H, K_{1}\right)$.

(51) $\operatorname{Evaluate}\left(H_{1} \wedge H_{2}, K_{1}\right)=\operatorname{Evaluate}\left(H_{1}, K_{1}\right) \wedge \operatorname{Evaluate}\left(H_{2}, K_{1}\right)$.

(52) Evaluate $\left(H_{1} \vee H_{2}, K_{1}\right)=$ Evaluate $\left(H_{1}, K_{1}\right) \vee$ Evaluate $\left(H_{2}, K_{1}\right)$.

(53) Evaluate $\left(\mathcal{X} H, K_{1}\right)=\mathcal{X}$ Evaluate $\left(H, K_{1}\right)$.

(54) Evaluate $\left(H_{1} \mathcal{U} H_{2}, K_{1}\right)=$ Evaluate $\left(H_{1}, K_{1}\right) \mathcal{U}$ Evaluate $\left(H_{2}, K_{1}\right)$.

(55) Evaluate $\left(H_{1} \mathcal{R} H_{2}, K_{1}\right)=\operatorname{Evaluate}\left(H_{1}, K_{1}\right) \mathcal{R}$ Evaluate $\left(H_{2}, K_{1}\right)$. 
Let $S$ be a non empty set. The infinite sequences of $S$ yielding a non empty set is defined by:

(Def. 40) The infinite sequences of $S=S^{\mathbb{N}}$.

Let $S$ be a non empty set and let $t$ be a sequence of $S$. The functor CastSeq $t$ yields an element of the infinite sequences of $S$ and is defined by:

(Def. 41) CastSeq $t=t$.

Let $S$ be a non empty set and let $t$ be a set. Let us assume that $t$ is an element of the infinite sequences of $S$. The functor $\operatorname{CastSeq}(t, S)$ yielding a sequence of $S$ is defined by:

(Def. 42) $\operatorname{CastSeq}(t, S)=t$.

Let $S$ be a non empty set, let $t$ be a sequence of $S$, and let $k$ be a natural number. The functor $\operatorname{Shift}(t, k)$ yielding a sequence of $S$ is defined as follows:

(Def. 43) For every natural number $n$ holds $(\operatorname{Shift}(t, k))(n)=t(n+k)$.

Let $S$ be a non empty set, let $t$ be a set, and let $k$ be a natural number. The functor $\operatorname{Shift}(t, k, S)$ yielding an element of the infinite sequences of $S$ is defined as follows:

(Def. 44) $\operatorname{Shift}(t, k, S)=$ CastSeq $\operatorname{Shift}(\operatorname{CastSeq}(t, S), k)$.

Let $S$ be a non empty set, let $t$ be an element of the infinite sequences of $S$, and let $k$ be a natural number. The functor $\operatorname{Shift}(t, k)$ yielding an element of the infinite sequences of $S$ is defined as follows:

(Def. 45) $\operatorname{Shift}(t, k)=\operatorname{Shift}(t, k, S)$.

Let $S$ be a non empty set and let $f$ be a set. The functor $\operatorname{Not}_{0}(f, S)$ yields an element of ModelSP (the infinite sequences of $S$ ) and is defined by the condition (Def. 46).

(Def. 46) Let $t$ be a set. Suppose $t \in$ the infinite sequences of $S$. Then $\neg$ Castboolean $(\operatorname{Fid}(f$, the infinite sequences of $S))(t)=$ true if and only if $\left(\operatorname{Fid}\left(\operatorname{Not}_{0}(f, S)\right.\right.$, the infinite sequences of $\left.\left.S\right)\right)(t)=$ true.

Let $S$ be a non empty set. The functor Not $S$ yielding a unary operation on ModelSP (the infinite sequences of $S$ ) is defined by:

(Def. 47) For every set $f$ such that $f \in$ ModelSP (the infinite sequences of $S$ ) holds $(\operatorname{Not} S)(f)=\operatorname{Not}_{0}(f, S)$.

Let $S$ be a non empty set, let $f$ be a function from the infinite sequences of $S$ into Boolean, and let $t$ be a set. The functor Next-univ $(t, f)$ yields an element of Boolean and is defined as follows:

(Def. 48) Next-univ $(t, f)=\left\{\begin{array}{c}\text { true, if } t \text { is an element of the infinite sequences } \\ \text { of } S \text { and } f(\operatorname{Shift}(t, 1, S))=\text { true, } \\ \text { false, otherwise. }\end{array}\right.$

Let $S$ be a non empty set and let $f$ be a set. The functor $\operatorname{Next}_{0}(f, S)$ yielding an element of ModelSP (the infinite sequences of $S$ ) is defined by the condition 
(Def. 49).

(Def. 49) Let $t$ be a set. Suppose $t \in$ the infinite sequences of $S$. Then $\operatorname{Next-univ}(t, \operatorname{Fid}(f$, the infinite sequences of $S))=$ true if and only if $\left(\operatorname{Fid}\left(\operatorname{Next}_{0}(f, S)\right.\right.$, the infinite sequences of $\left.\left.S\right)\right)(t)=$ true.

Let $S$ be a non empty set. The functor Next $S$ yields a unary operation on ModelSP (the infinite sequences of $S$ ) and is defined as follows:

(Def. 50) For every set $f$ such that $f \in$ ModelSP (the infinite sequences of $S$ ) holds $(\operatorname{Next} S)(f)=\operatorname{Next}_{0}(f, S)$.

Let $S$ be a non empty set and let $f, g$ be sets. The functor $\operatorname{And}_{0}(f, g, S)$ yields an element of ModelSP (the infinite sequences of $S$ ) and is defined by the condition (Def. 51).

(Def. 51) Let $t$ be a set. Suppose $t \in$ the infinite sequences of $S$. Then Castboolean $(\operatorname{Fid}(f$, the infinite sequences of $S))(t) \wedge$ Castboolean $(\operatorname{Fid}(g$, the infinite sequences of $S))(t)=$ true if and only if $\left(\operatorname{Fid}\left(\operatorname{And}_{0}(f, g, S)\right.\right.$, the infinite sequences of $S))(t)=$ true.

Let $S$ be a non empty set. The functor And $S$ yielding a binary operation on ModelSP (the infinite sequences of $S$ ) is defined by the condition (Def. 52).

(Def. 52) Let $f, g$ be sets. Suppose $f \in \operatorname{ModelSP}$ (the infinite sequences of $S$ ) and $g \in$ ModelSP (the infinite sequences of $S$ ). Then $($ And $S)(f, g)=$ $\operatorname{And}_{0}(f, g, S)$.

Let $S$ be a non empty set, let $f, g$ be functions from the infinite sequences of $S$ into Boolean, and let $t$ be a set. The functor Until-univ $(t, f, g, S)$ yields an element of Boolean and is defined as follows:

(Def. 53) Until-univ $(t, f, g, S)=\left\{\begin{array}{l}\text { such that for every natural number } j \\ \text { such that } j<m \text { holds } f(\operatorname{Shift}(t, j, S))= \\ \text { true and } g(\operatorname{Shift}(t, m, S))=\text { true, } \\ \text { false, otherwise. }\end{array}\right.$

Let $S$ be a non empty set and let $f, g$ be sets. The functor $\operatorname{Until}_{0}(f, g, S)$ yields an element of ModelSP (the infinite sequences of $S$ ) and is defined by the condition (Def. 54).

(Def. 54) Let $t$ be a set. Suppose $t \in$ the infinite sequences of $S$. Then $\operatorname{Until-univ}(t, \operatorname{Fid}(f$, the infinite sequences of $S), \operatorname{Fid}(g$, the infinite sequences of $S), S)=$ true if and only if $\left(\operatorname{Fid}\left(\operatorname{Until}_{0}(f, g, S)\right.\right.$, the infinite sequences of $S))(t)=$ true.

Let $S$ be a non empty set. The functor Until $S$ yielding a binary operation on ModelSP (the infinite sequences of $S$ ) is defined by the condition (Def. 55).

(Def. 55) Let $f, g$ be sets. Suppose $f \in \operatorname{ModelSP}$ (the infinite sequences of $S$ ) and $g \in$ ModelSP (the infinite sequences of $S$ ). Then $(\operatorname{Until} S)(f, g)=$ 
$\operatorname{Until}_{0}(f, g, S)$.

Let $S$ be a non empty set. The functor $\vee_{S}$ yields a binary operation on ModelSP (the infinite sequences of $S$ ) and is defined by the condition (Def. 56).

(Def. 56) Let $f, g$ be sets. Suppose $f \in$ ModelSP (the infinite sequences of $S$ ) and $g \in \operatorname{ModelSP}$ (the infinite sequences of $S$ ). Then $\vee_{S}(f, g)=$ $(\operatorname{Not} S)(($ And $S)((\operatorname{Not} S)(f),(\operatorname{Not} S)(g)))$.

The functor Release $S$ yields a binary operation on ModelSP (the infinite sequences of $S$ ) and is defined by the condition (Def. 57).

(Def. 57) Let $f, g$ be sets. Suppose $f \in \operatorname{ModelSP}$ (the infinite sequences of $S$ ) and $g \in$ ModelSP (the infinite sequences of $S$ ). Then (Release $S)(f, g)=$ $(\operatorname{Not} S)((\operatorname{Until} S)((\operatorname{Not} S)(f),(\operatorname{Not} S)(g)))$.

Let $S$ be a non empty set and let $B_{1}$ be a non empty subset of ModelSP (the infinite sequences of $S$ ). The functor $\operatorname{Model}_{\text {LTL }}\left(S, B_{1}\right)$ yields an LTL-model structure and is defined as follows:

(Def. 58) $\operatorname{Model}_{\mathrm{LTL}}\left(S, B_{1}\right)=\left\langle\operatorname{ModelSP}\left(\right.\right.$ the infinite sequences of $S$ ), $B_{1}$, And $S$, $\vee_{S}$, Not $S$, Next $S$, Until $S$, Release $\left.S\right\rangle$.

In the sequel $B_{1}$ denotes a non empty subset of ModelSP (the infinite sequences of $S$ ), $t$ denotes an element of the infinite sequences of $S$, and $f, g$ denote assignations of $\operatorname{Model}_{\mathrm{LTL}}\left(S, B_{1}\right)$.

Let $S$ be a non empty set, let $B_{1}$ be a non empty subset of ModelSP (the infinite sequences of $S$ ), let $t$ be an element of the infinite sequences of $S$, and let $f$ be an assignation of $\operatorname{Model}_{\text {LTL }}\left(S, B_{1}\right)$. The predicate $t=f$ is defined by:

(Def. 59) $\quad(\operatorname{Fid}(f$, the infinite sequences of $S))(t)=$ true.

Let $S$ be a non empty set, let $B_{1}$ be a non empty subset of ModelSP (the infinite sequences of $S$ ), let $t$ be an element of the infinite sequences of $S$, and let $f$ be an assignation of $\operatorname{Model}_{\mathrm{LTL}}\left(S, B_{1}\right)$. We introduce $t \not \models f$ as an antonym of $t \models f$.

The following propositions are true:

(56) $\quad f \vee g=\neg(\neg f \wedge \neg g)$ and $f \mathcal{R} g=\neg(\neg f \mathcal{U} \neg g)$.

(57) $t \models \neg f$ iff $t \not \models f$.

(58) $t \models f \wedge g$ iff $t \models f$ and $t \models g$.

(59) $t=\mathcal{X} f$ iff $\operatorname{Shift}(t, 1) \models f$.

(60) $t \models f \mathcal{U} g$ if and only if there exists a natural number $m$ such that for every natural number $j$ such that $j<m$ holds $\operatorname{Shift}(t, j) \models f$ and $\operatorname{Shift}(t, m) \models g$.

(61) $t \models f \vee g$ iff $t \models f$ or $t \models g$.

(62) $t \models f \mathcal{R} g$ if and only if for every natural number $m$ such that for every natural number $j$ such that $j<m$ holds $\operatorname{Shift}(t, j) \models \neg f$ holds $\operatorname{Shift}(t, m) \models g$. 
The non empty set AtomicFamily is defined as follows:

(Def. 60) AtomicFamily $=2^{\text {atomic }_{\text {LTL }}}$.

Let $a, t$ be sets. The functor AtomicFunc $(a, t)$ yielding an element of Boolean is defined as follows:

(Def. 61) AtomicFunc $(a, t)=\left\{\begin{array}{c}\text { true, if } t \in \text { the infinite sequences of AtomicFamily } \\ \text { and } a \in(\text { CastSeq }(t, \text { AtomicFamily }))(0), \\ \text { false, otherwise. }\end{array}\right.$

Let $a$ be a set. The functor AtomicAsgn $a$ yields an element of ModelSP (the infinite sequences of AtomicFamily) and is defined by:

(Def. 62) For every set $t$ such that $t \in$ the infinite sequences of AtomicFamily holds $(\operatorname{Fid}($ AtomicAsgn $a$, the infinite sequences of AtomicFamily $))(t)=$ AtomicFunc $(a, t)$.

The non empty subset AtomicBasicAsgn of ModelSP (the infinite sequences of AtomicFamily) is defined by:

(Def. 63) AtomicBasicAsgn $=\{x \in \operatorname{ModelSP}$ (the infinite sequences of AtomicFamily): $\bigvee_{a: \text { set }} x=$ AtomicAsgn $\left.a\right\}$.

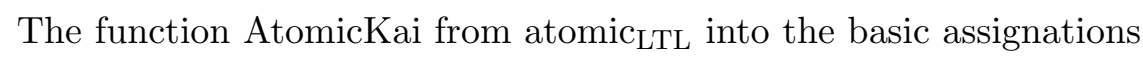
of Model $_{\mathrm{LTL}}$ (AtomicFamily, AtomicBasicAsgn) is defined as follows:

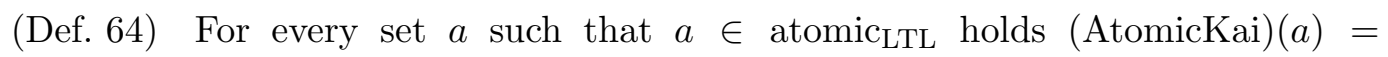
AtomicAsgn $a$.

Let $r$ be an element of the infinite sequences of AtomicFamily and let $H$ be an LTL-formula. The predicate $r \models H$ is defined by:

(Def. 65) $r \models \operatorname{Evaluate}(H$, AtomicKai).

Let $r$ be an element of the infinite sequences of AtomicFamily and let $H$ be an LTL-formula. We introduce $r \not \models H$ as an antonym of $r=H$.

Let $r$ be an element of the infinite sequences of AtomicFamily and let $W$ be a subset of $\mathrm{WFF}_{\mathrm{LTL}}$. The predicate $r \models W$ is defined by:

(Def. 66) For every LTL-formula $H$ such that $H \in W$ holds $r \models H$.

Let $r$ be an element of the infinite sequences of AtomicFamily and let $W$ be a subset of $\mathrm{WFF}_{\mathrm{LTL}}$. We introduce $r \not \models W$ as an antonym of $r \models W$.

Let $W$ be a subset of $\mathrm{WFF}_{\mathrm{LTL}}$. The functor $\mathcal{X} W$ yielding a subset of $\mathrm{WFF}_{\mathrm{LTL}}$ is defined as follows:

(Def. 67$) \mathcal{X} W=\left\{x ; x\right.$ ranges over LTL-formulae: $\bigvee_{u \text { :LTL-formula }}(u \in W \wedge x=$ $\mathcal{X} u)\}$.

In the sequel $r$ denotes an element of the infinite sequences of AtomicFamily.

We now state a number of propositions:

(63) If $H$ is atomic, then $r \models H$ iff $H \in(\operatorname{CastSeq}(r, \operatorname{AtomicFamily}))(0)$.

(64) $r \models \neg H$ iff $r \not \models H$.

(65) $r \models H_{1} \wedge H_{2}$ iff $r \models H_{1}$ and $r \models H_{2}$. 
(66) $r \models H_{1} \vee H_{2}$ iff $r \models H_{1}$ or $r \models H_{2}$.

(67) $r \models \mathcal{X} H$ iff $\operatorname{Shift}(r, 1) \models H$.

(68) $r \models H_{1} \mathcal{U} H_{2}$ if and only if there exists a natural number $m$ such that for every natural number $j$ such that $j<m$ holds $\operatorname{Shift}(r, j) \models H_{1}$ and $\operatorname{Shift}(r, m) \models H_{2}$.

(69) $r \models H_{1} \mathcal{R} H_{2}$ if and only if for every natural number $m$ such that for every natural number $j$ such that $j<m$ holds $\operatorname{Shift}(r, j) \models \neg H_{1}$ holds $\operatorname{Shift}(r, m) \models H_{2}$.

(70) $r \models \neg\left(H_{1} \vee H_{2}\right)$ iff $r \models \neg H_{1} \wedge \neg H_{2}$.

(71) $r \models \neg\left(H_{1} \wedge H_{2}\right)$ iff $r \models \neg H_{1} \vee \neg H_{2}$.

(72) $\quad r \models H_{1} \mathcal{R} H_{2}$ iff $r=\neg\left(\neg H_{1} \mathcal{U} \neg H_{2}\right)$.

(73) $r \not \models \neg$ iff $r \mid=H$.

(74) $r \models \mathcal{X} \neg H$ iff $r \models \neg \mathcal{X} H$.

(75) $r \models H_{1} \mathcal{U} H_{2}$ iff $r=H_{2} \vee H_{1} \wedge \mathcal{X}\left(H_{1} \mathcal{U} H_{2}\right)$.

(76) $r \models H_{1} \mathcal{R} H_{2}$ iff $r \models H_{1} \wedge H_{2} \vee H_{2} \wedge \mathcal{X}\left(H_{1} \mathcal{R} H_{2}\right)$.

In the sequel $W$ is a subset of $\mathrm{WFF}_{\mathrm{LTL}}$.

One can prove the following propositions:

(77) $r \models \mathcal{X} W$ iff $\operatorname{Shift}(r, 1) \models W$.

(78)(i) If $H$ is atomic, then $H$ is not negative and $H$ is not conjunctive and $H$ is not disjunctive and $H$ does not have next operator and $H$ does not have until operator and $H$ does not have release operator,

(ii) if $H$ is negative, then $H$ is not atomic and $H$ is not conjunctive and $H$ is not disjunctive and $H$ does not have next operator and $H$ does not have until operator and $H$ does not have release operator,

(iii) if $H$ is conjunctive, then $H$ is not atomic and $H$ is not negative and $H$ is not disjunctive and $H$ does not have next operator and $H$ does not have until operator and $H$ does not have release operator,

(iv) if $H$ is disjunctive, then $H$ is not atomic and $H$ is not negative and $H$ is not conjunctive and $H$ does not have next operator and $H$ does not have until operator and $H$ does not have release operator,

(v) if $H$ has next operator, then $H$ is not atomic and $H$ is not negative and $H$ is not conjunctive and $H$ is not disjunctive and $H$ does not have until operator and $H$ does not have release operator,

(vi) if $H$ has until operator, then $H$ is not atomic and $H$ is not negative and $H$ is not conjunctive and $H$ is not disjunctive and $H$ does not have next operator and $H$ does not have release operator, and

(vii) if $H$ has release operator, then $H$ is not atomic and $H$ is not negative and $H$ is not conjunctive and $H$ is not disjunctive and $H$ does not have next operator and $H$ does not have until operator.

(79) For every element $t$ of the infinite sequences of $S$ holds Shift $(t, 0)=t$. 
(80) For every element $s_{1}$ of the infinite sequences of $S$ holds $\operatorname{Shift}\left(\operatorname{Shift}\left(s_{1}, k\right), n\right)=\operatorname{Shift}\left(s_{1}, n+k\right)$.

(81) For every sequence $s_{1}$ of $S$ holds CastSeq(CastSeq $\left.s_{1}, S\right)=s_{1}$.

(82) For every element $s_{1}$ of the infinite sequences of $S$ holds CastSeq $\operatorname{CastSeq}\left(s_{1}, S\right)=s_{1}$.

(83) If $H, \neg H \in W$, then $r \not \models W$.

\section{REFERENCES}

[1] Grzegorz Bancerek. The fundamental properties of natural numbers. Formalized Mathematics, 1(1):41-46, 1990.

[2] Grzegorz Bancerek. A model of ZF set theory language. Formalized Mathematics, 1(1):131-145, 1990

[3] Grzegorz Bancerek. Models and satisfiability. Formalized Mathematics, 1(1):191-199, 1990.

[4] Grzegorz Bancerek and Krzysztof Hryniewiecki. Segments of natural numbers and finite sequences. Formalized Mathematics, 1(1):107-114, 1990.

[5] Czesław Byliński. Binary operations. Formalized Mathematics, 1(1):175-180, 1990.

[6] Czesław Byliński. Functions and their basic properties. Formalized Mathematics, 1(1):5565, 1990.

[7] Czesław Byliński. Functions from a set to a set. Formalized Mathematics, 1(1):153-164, 1990.

[8] Czesław Byliński. Some basic properties of sets. Formalized Mathematics, 1(1):47-53, 1990.

[9] E. M. Clarke, O. Grumberg, and D. Peled. Model Checking. MIT Press, 2000.

[10] Kazuhisa Ishida. Model checking. Part I. Formalized Mathematics, 14(4):171-186, 2006.

[11] Zinaida Trybulec. Properties of subsets. Formalized Mathematics, 1(1):67-71, 1990.

[12] Edmund Woronowicz. Many-argument relations. Formalized Mathematics, 1(4):733-737, 1990. 